\title{
Research on the Steel for Oil and Gas Pipelines in Sour Environment
}

\author{
Xiaodong Shao ${ }^{1,2 *}$ \\ ${ }^{1}$ State Key Laboratory for Performance and Structure Safety of Petroleum Tubular Goods and Equipment Materials, \\ No. 89 Jinyeer Road, Xi'an 710077, China \\ ${ }^{2}$ CNPC Tubular Goods Research Institute, No. 89 Jinyeer Road, Xi’an 710077, China
}

\begin{abstract}
In recent years, there are more and more oil and gas fields containing $\mathrm{H}_{2} \mathrm{~S}$ corrosive media, and it is imperative to develop oil and gas transmission pipes in acidic environment. The research progress of steel for oil and gas pipelines in acidic environment is reviewed from the aspects of $\mathrm{H}_{2} \mathrm{~S}$ corrosion mechanism and influencing factors in pipeline service under acidic service conditions. At the same time, the development status of sour-resistance corrosion oil and gas transmission steel tubes at home and abroad is introduced. Pipeline steel is further explored in terms of SSCC-resistance research and improvement of corresponding standards.
\end{abstract}

\section{Introduction}

Oil and gas pipeline transportation is characterized by high efficiency, economy and safety, and is the main form of long-distance transportation of oil and gas [1]. The development of pipelines tends to be large-caliber and high-pressure, the service conditions of pipelines are becoming more and more demanding, the transportation medium is complex, and many pipelines need to cross densely populated areas or deserts, cold regions, etc., which puts forward the performance of pipelines. High technical requirements, especially the requirements of corrosion resistance, Academician Li Helin pointed out that anti-hydrogen induced cracking (HIC) pipeline steel and steel pipe is one of the development directions of high performance oil and gas transmission steel pipe. Corrosion is a key factor affecting the reliability and service life of pipeline transportation systems. It can not only cause perforation, but also cause oil and gas leakage. It may also cause an explosion, threaten personal safety, pollute the environment, and the consequences are extremely serious. According to the National Transportation Safety Administration, $74 \%$ of the leaks in the US gas pipelines and gas gathering pipelines are caused by corrosion, and $\mathrm{H}_{2} \mathrm{~S}$ corrosion is one of the main forms of pipeline corrosion. According to the American Society of Corrosion Engineers (NACE), in natural gas containing water and $\mathrm{H}_{2} \mathrm{~S}$, when the partial pressure of $\mathrm{H}_{2} \mathrm{~S}$ in the gas is equal to or greater than $0.0003 \mathrm{MPa}$, it is called an acidic environment.

Since the 1970s, the development conditions of oil and natural gas have changed significantly in various countries. At present, although the purification process has been carried out before the natural gas transmission, the natural gas to be transported into the gas pipeline does not meet the requirements of dry and purified natural gas. $\mathrm{H}_{2} \mathrm{~S}$ in the pipeline. In-pipe corrosion caused by the presence of water is still inevitable. In recent years, accidents caused by pipe $\mathrm{H}_{2} \mathrm{~S}$ corrosion have occurred, resulting in huge economic losses and environmental pollution. Therefore, solving or mitigating $\mathrm{H}_{2} \mathrm{~S}$ corrosion of oil and gas pipelines has very important economic and social benefits. According to the development needs of Chinese oil and gas industry, it is imperative to develop Chinese own acidic service conditions to transport steel pipes. Foreign countries have started research in this area earlier, and there are specialized agencies to study the transportation of steel pipes in an acidic environment. Although domestic work in this area started late, it also carried out a series of research work. The author mainly reviews the research progress of steel for acid and oil pipelines from the corrosion mechanism and influencing factors of $\mathrm{H}_{2} \mathrm{~S}$ in acidic environment pipelines, and introduces the development of oil and gas pipelines in acidic environment at home and abroad. Research direction.

\section{$2 \mathrm{H}_{2} \mathrm{~S}$ corrosion mechanism of pipeline steel}

The corrosion of pipeline steel in acidic environment mainly includes hydrogen induced cracking and sulfide stress corrosion cracking (SSCC). At present, the research on $\mathrm{H}_{2} \mathrm{~S}$ corrosion of pipeline steel is mainly focused on HIC. Pipeline steel in the oil-rich environment rich in $\mathrm{H}_{2} \mathrm{~S}$ in the state of no stress or nontensile stress, the hydrogen generated by the corrosion into the steel to form a crack called HIC, generally refers to hydrogen-induced bubbling (surface crack) and hydrogen-induced step cracking ( Internal crack). SSCC

\footnotetext{
*Corresponding author: shaoxd@163.com
} 
means that the hydrogen atoms generated by $\mathrm{H}_{2} \mathrm{~S}$ penetrate into the interior of the steel, dissolve in the crystal lattice, cause brittleness, and form cracks under the action of external tensile stress or residual stress. SSCC is in sensitive materials, acidic environment and stretching. The three conditions of stress occur together [2]. When transporting acidic oil and gas, the inside of the pipeline is exposed to $\mathrm{H}_{2} \mathrm{~S}$. Under the joint action of stress and $\mathrm{H}_{2} \mathrm{~S}$ and other corrosive media, SSCC often occurs in the pipeline.

\section{Research on the HIC of pipeline steel}

\subsection{Influence of the environment on HIC}

The results show that when the partial pressure of $\mathrm{H}_{2} \mathrm{~S}$ is $0.1 \sim 0.5 \mathrm{MPa}$, the corresponding hydrogen permeability is higher, which means that the sensitivity of HIC is greater [3]. As the $\mathrm{pH}$ decreases, the HIC sensitivity of the pipeline steel also increases. When $\mathrm{CO}_{2}$ is dissolved in water to form carbonic acid, hydrogen ions are released, which lowers the $\mathrm{pH}$ of the environment and increases the sensitivity of $\mathrm{HIC}$. $\mathrm{Cl}^{-}$accelerates the corrosion rate in the range of $\mathrm{pH} 3.5 \sim 4.5$, and the HIC sensitivity increases [4]. When the temperature is around $30^{\circ} \mathrm{C}$, the additive effect of hydrogen adsorption and diffusion is the largest, that is, the HIC sensitivity is the strongest, below $30^{\circ} \mathrm{C}$, the hydrogen diffusion rate and activity decrease, and it is difficult to aggregate active hydrogen above $30^{\circ} \mathrm{C}$.

\subsection{Influence of composition and structure on HIC}

The influence of pipeline steel composition and structure on HIC is the most in-depth study, and the results are also the most, which can be summarized into three aspects.

(1) Chemical composition. The elements that are more sensitive to HIC are carbon, manganese, sulfur, phosphorus, calcium, copper, and molybdenum. As the carbon content increases, the HIC sensitivity increases, and the increase in carbon content and carbon equivalent in the pipeline steel causes the steel to form the martensite structure most sensitive to hydrogen bubbling during hot rolling, thus reducing the carbon content and carbon equivalent. It can improve the $\mathrm{H}_{2} \mathrm{~S}$-resistance corrosion performance of pipeline steel. Sulfur can promote the occurrence of HIC, which is the most easily nucleated site of HIC with manganese-generated $\mathrm{MnS}$. Baosteel's research indicates that the addition of calcium can change the form of sulfide inclusions, making it a dispersed spheroid, thereby improving the HICresistance ability of steel. The test found that the best effect is when the calcium-sulfur content ratio is greater than 1.5. Adding an appropriate amount of manganese to the pipeline steel can improve the hardenability of the steel, cause solid solution strengthening, and compensate for the decrease in strength caused by low carbon. The segregation of manganese and phosphorus can cause the formation of band-like structures sensitive to HIC, thus increasing the manganese content., will lead to more banded tissue generation, which increases HIC sensitivity. The effect of copper on HIC is obvious. In NACE B solution, HIC sensitivity decreases significantly with increasing copper content. However, in the $\mathrm{H}_{2} \mathrm{~S}$ environment with $\mathrm{pH}<4.5$, the passivation film of copper is no longer formed, and at this time, the copper prevents the effect of HIC from disappearing. The addition of molybdenum can lower the phase transition temperature, inhibit the formation of massive ferrite, promote the transformation of acicular ferrite, improve the strength of steel, reduce the ductile-brittle transition temperature, and improve the HIC-resistance ability. The addition of microalloying elements such as bismuth, vanadium and titanium to pipeline steel can effectively prevent the growth of austenite grains, refine grains and enhance the corrosion resistance of pipeline steels [5,6]. Due to the influence of chemical composition on the HIC resistance of pipeline steel, the chemical composition of different steel grade HICresistance oil and gas transmission steel pipes has clear technical requirements in ISO 3183.

(2) Microstructure. Sodium thiocyanate was selected as the chromogenic reagent in the proposed method. The effect of the chromogenic reagent was examined by measuring the absorbance of solution containing certain amounts of molybdenum and variable amounts of sodium thiocyanate. It was found that $10 \mathrm{ml}$ of $100.0 \mathrm{mg} \cdot \mathrm{mL}^{-1}$ sodium thiocyanate solution sufficed to complex the amounts of molybdenum taken, with higher concentrations the absorbance was essentially constant. Ten millilitres of $100.0 \mathrm{mg} \cdot \mathrm{mL}^{-1}$ sodium thiocyanate solution were recommended as a suitable amount of chromogenic reagent. The thermodynamically balanced and stable fine grain structure is the ideal structure for HIC-resistance. For medium and low-strength pipeline steels, HIC is prone to low temperature conversion of manganese, phosphorus and other elements in the banded pearlite structure and the center of the plate thickness. In particular, in order to improve the strength of pipeline steel, the addition of many alloying elements promotes the formation of low temperature conversion microstructure, which provides a convenient place for the occurrence of HIC [7]. With the increase of the microstructure of steel, the HIC-resistance performance decreases gradually, and the HIC-resistance performance decreases with the increase of martensite structure. With the increase of the band structure level, the HICresistance performance of pipeline steel is gradually reduced. Yin Guanghong et al found that the HICresistance performance of pipeline steel will be significantly enhanced after normalizing heat treatment.

(3) Non-metallic inclusions. The morphology and distribution of non-metallic inclusions affect the HIC resistance of pipeline steel. The non-metallic inclusion interface is a strong hydrogen trap [8]. On the interface between the pipeline steel and $\mathrm{H}_{2} \mathrm{~S}$, the hydrogen ions generated by the electrochemical reaction are trapped in the hydrogen trap after the electrons are converted into hydrogen atoms at the anode. When the hydrogen pressure rises to a certain value, it is in the non-metal. Cracks occur at the interface of the inclusions, and 
plastic deformation occurs at the crack tip. In pipeline steel, the higher the content of non-metallic inclusions, the smaller the threshold value of hydrogen concentration required for HIC, that is, the stronger the HIC sensitivity [9]. In addition, in the same pipeline steel, the coarser the grain, the worse the HIC-resistance performance [10]. Some scholars have pointed out that the hardness of the material also has a great influence on HIC. In the medium and low strength steel, the higher the strength and the greater the hardness, the greater the HIC sensitivity.

\section{Research on the SSCC of pipeline steel}

\subsection{Influence of the environment on SSCC}

The lower the $\mathrm{pH}$ of the environment, the greater the hydrogen concentration and the stronger the sensitivity of the SSCC. At the same time, the amount of hydrogen permeation in the pipeline steel increases with the increase of $\mathrm{H}_{2} \mathrm{~S}$ concentration, and the SSCC sensitivity of the pipeline steel is greater [11], the high-strength steel SSCC has a most sensitive temperature, near this temperature, occurs The SSCC is the most likely.

\subsection{Influence of composition and structure on SSCC}

The composition and organization of pipeline steel is another major factor affecting pipeline steel SSCC.

(1) Chemical composition. Regarding the influence of carbon, different scholars have different opinions, but regardless of the explanation, the lower the carbon content, the lower the sensitivity of the SSCC of pipeline steel. Du Shiyu and other research through the domestic X70 pipeline steel, pointed out that the main chemical elements affecting its SSCC are manganese and phosphorus content, not carbon content. Molybdenum is a beneficial element, especially in the case where high strength of the material is required, a small amount of molybdenum is added to form carbides, so that the amount of solid solution carbon is reduced, and thus the performance against SSCC is improved. The role of chromium has two different perspectives, but it is beneficial for improving general corrosion resistance. The addition of titanium and boron improves the hardenability and also has a certain effect on improving the resistance to SSCC $[12,13]$. Aluminum is a beneficial element, especially for materials that add both chromium and aluminum.

(2) Microstructure. The microstructure of pipeline steel has a great influence on the sensitivity of SSCC. Martensite is generally considered to be more sensitive than pearlite and austenite. Xie Guangyu et al studied the SSCC behavior of X70 grade pipeline steel with different microstructures by three-point bending method. The results show that the uniform fine acicular ferrite microstructure has excellent SSCC resistance. Zhao Mingchun et al studied the SSCC-resistance behavior of different microstructure pipeline steels. The results showed that the acicular ferrite microstructure had the best performance against SSCC, the ultrafine ferrite structure was the second, and the microstructure of ferrite pearlite. Worst. Mansour et al. [14] studied the SSCC behavior of X100 pipeline steel and pointed out that with the increase of the microstructure of steel, the performance of SSCC decreased. In general, the higher the strength level of pipeline steel, the greater the sensitivity to SSCC, the highest hardness value of SSCC does not occur between 20 27HRC, the higher the hardness, the lower the critical stress value and the fracture time. In addition, due to a series of physical and chemical changes in the weld during the welding process and some weld defects, the ability of the welded joint to resist SSCC is reduced relative to the base metal. The SSCC resistance of the pipeline steel can be improved by eliminating the residual stress annealing treatment. Tao Yongqi et al. showed that different weld matching in $\mathrm{H}_{2} \mathrm{~S}$-containing media resulted in different corrosion resistance of pipeline steels, with the increase of cold deformation, the resistance of pipeline steel to SSCC was reduced.

\section{Development of $\mathrm{H}_{2} \mathrm{~S}$-resistance corrosion pipeline steel}

Some scholars believe that there is a difference and a relationship between the HIC and SSCC of pipeline steel. It can be considered that the SSCC crack of pipeline steel is a hydrogen-induced cracking stress corrosion. If the HIC of the pipeline steel can be controlled, the necessary conditions for the occurrence of SSCC $\mathrm{H}_{2} \mathrm{~S}$ corrosion or material sensitivity are not available, so that the SSCC crack of the pipeline steel can be controlled. Therefore, the development and evaluation of $\mathrm{H}_{2} \mathrm{~S}$ resistance corrosion transport steel pipes at home and abroad mainly focus on the HIC-resistance performance of steel. Pipeline steels used in acidic environments are characterized by high toughness and resistance to HIC properties that depend on the high purity of the steel.

In order to meet the strength, weldability and manufacturability, it is very important to control the process parameters of the HIC pipeline steel, such as composition design, smelting, continuous casting, rolling, etc., in order to ensure a uniform microstructure. Through the research on the corrosion mechanism and influencing factors of $\mathrm{H}_{2} \mathrm{~S}$, the measures to improve the HIC resistance of pipeline steel have formed a consensus, using concentrate and high-efficiency iron pretreatment and re-refining outside the furnace to improve the purity of steel. Calcium treatment is carried out while reducing sulfur content, electromagnetic stirring is carried out during steel smelting and continuous casting, light reduction technology is adopted in continuous casting process, multi-stage controlled rolling and forced accelerated cooling process are used to limit band structure, reduce carbon content, control manganese content, add copper and nickel, fully refine the grains by microalloying and controlled rolling processes. 
At present, the general method adopted for the evaluation of steel HIC-resistance ability is the standard test method NACE TM0284 issued by NACE, including three evaluations of crack sensitivity rate (CSR), crack length ratio (CLR) and crack thickness ratio (CTR). parameter. Since the 1990s, the HIC-resistance steel pipes supplied by foreign countries are mainly concentrated in the X65 steel grade. The HIC-resistance oil and gas transmission steel pipes produced by the European steel pipe companies have accounted for more than $30 \%$ of their sales, the X70 steel pipes with HICresistance have also been successfully developed. It is used on a pipeline in Mexico. Some countries have developed X80 steel pipes resistant to HIC, but they have not been supplied in bulk and are still under further study. Sumitomo Metal Industries Co., Ltd. has developed X80 steel grade pipe steel with excellent HIC resistance and steel pipe made of this steel. For X70 and below steel grade HIC-resistance steel pipes, there are clear requirements for HIC resistance evaluation in the world: $\mathrm{CSR} \leq 2 \%, \mathrm{CLR} \leq 15 \%, \mathrm{CTR} \leq 5 \%$. Although the research and development of domestic HIC-resistance pipeline steel has just started, it has also achieved some results. According to the above design principles, China has developed HIC-resistance pipeline steel including X80 steel grade. Pangang Group Chengdu Iron and Steel Co., Ltd. is at the domestic leading level in the research and development of anti-acid corrosion seamless steel tubes, with mass production capacity. The submarine pipelines developed by the company are resistant to acid corrosion X56, X60 and X65 steel grade series seamless steel tubes. The microalloyed component design and the corresponding process technology route solve the technical problems of smelting, rolling and heat treatment processes, and the product indicators meet the requirements of the corresponding standards. Baoji Petroleum Pipe Co., Ltd. also developed a spiral submerged arc welded pipe for X52 $\mathrm{H}_{2} \mathrm{~S}$-resistance corrosion gas gathering pipeline. Laiwu Iron \& Steel Co., Ltd. has developed a manufacturing method for HICresistance pipeline steel. The physical and chemical properties of the product have reached X46 and X52 steel grades, which can realize multi-purpose steel. The X52, X60 and X65 grade HIC pipeline steels developed by Baosteel have high strength and high toughness and good HIC resistance. The main properties are listed in Table 1. X65 grade pipeline steel also has better resistance to dynamic tearing. Performance and higher impact toughness, which helps to ensure the safety of high pressure gas pipeline operation. Wugang developed a large-thickness and high-performance HIC-resistance steel for the Sichuan Puguang gas field, and formed mass production, which successfully replaced imported products. Baosteel is at the domestic leading level in the development of X80 steel grade HIC-resistance pipeline steel. It adopts lower carbon, phosphorus and sulfur content in the composition design, and at the same time, in order to make up for the strength reduction caused by the decrease of carbon content, proper addition is beneficial to the HIC-resistance performance. Alloying elements such as copper and nickel. In order to obtain a microstructure with less segregation, increase the niobium content and low carbon content, $\mathrm{NbC}$ particles are obtained, the microstructure is refined to increase the recrystallization termination temperature, and the tissue segregation is reduced, thereby ensuring strength and toughness and ensuring HIC-resistance performance. The method also adopts the pipeline steel composition and process design with "ultra-low carbon acicular ferrite" as the structural feature to eliminate the banded pearlite structure in the steel to ensure the HIC-resistance performance of the pipeline steel. The HIC-resistance performance of the product was evaluated as $\mathrm{CSR} \leq 0.01 \%, \mathrm{CLR} \leq 0.09 \%$, and $\mathrm{CTR} \leq 0.02 \%$.

\section{Conclusions}

With the advancement of metallurgical technology, after years of development and production, the output of pipeline steel in China has increased year by year.

Table 1. Properties of HIC-resistance pipeline steel produced by Baosteel

\begin{tabular}{|c|c|c|c|c|c|c|c|c|}
\hline \multirow[b]{2}{*}{ Grade } & \multirow[b]{2}{*}{$\begin{array}{c}\text { Specification } \\
\mathrm{mm}\end{array}$} & \multirow[b]{2}{*}{$\begin{array}{l}\mathrm{R}_{\mathrm{t} 0.5} \\
\mathrm{MPa}\end{array}$} & \multirow[b]{2}{*}{$\begin{array}{c}\mathrm{R}_{\mathrm{m}} \\
\mathrm{MPa}\end{array}$} & \multirow[b]{2}{*}{$\begin{array}{l}\mathrm{A} \\
\%\end{array}$} & \multicolumn{3}{|c|}{$A_{\mathrm{kV}}\left(-30^{\circ} \mathrm{C}\right) / \mathrm{J}$} & \multirow[b]{2}{*}{$\begin{array}{c}\text { NACE TM0284 } \\
\text { A Solution }\end{array}$} \\
\hline & & & & & Tube & $\begin{array}{c}\text { Heat } \\
\text { affected } \\
\text { zone } \\
\end{array}$ & Weld & \\
\hline$\times 52$ & $Ф 508 \times 11.9$ & 425 & 546 & 44 & 375 & 239 & 198 & No crack \\
\hline$x 60$ & $\Phi 711 \times 8.0$ & 476 & 598 & 41 & 208 & 180 & 178 & No crack \\
\hline$x 65$ & $\Phi 508 \times 8.7$ & 479 & 599 & 39 & 388 & 196 & 192 & No crack \\
\hline
\end{tabular}

The products have been applied to a series of major long-distance oil and gas pipeline projects such as the West-East Gas Pipeline Project. It has been able to mass produce X65 pipeline steel resistant to HIC, X80 steel grade HIC-resistance pipeline steel has also been developed. However, it must be recognized that the research and development of Chinese HIC-resistance pipeline steel has just started, and there is still a certain gap with the advanced level of foreign countries.

With Chinese energy structure adjustment and environmental protection demand, the demand for natural gas has been increasing, and natural gas development conditions have also undergone significant changes. Acidic oil and gas fields have been rapidly 
developed. Therefore, in the future, it is necessary to further strengthen the research and development of steel for oil and gas pipelines in acidic environments. First of all, although there is a relationship between HIC and SSCC of pipeline steel, the research on pipeline steel HIC can not completely replace the research on SSCC performance of pipeline steel. At present, there are some researches on the influencing factors of SSCC, but the lack of SSCC is unique. The study of the key factors affecting tensile stress should strengthen the research work in this area and further develop the development of targeted SSCC-resistance pipeline steel. Secondly, in order to better study the HIC generated by pipeline steel in the actual acidic environment, the standard evaluation test of NACE TM0284 should be extended and improved through relevant experimental research. NACE has published the corresponding standard test method NACE TM0177 for evaluation. The material's ability to resist SSCC, but there is no clear technical parameter requirement in the world to measure the anti-SSCC ability of steel, and should be further explored and improved. Finally, there are few studies on the influence of $\mathrm{H}_{2} \mathrm{~S}$ corrosion products on the stress corrosion rate of hydrogen sulfide, and the $\mathrm{H}_{2} \mathrm{~S}$ corrosion products have some influence on the HIC crack and SSCC crack growth rate, and then the crack growth rate model can be predicted. And the establishment of difficulties.

\section{Acknowledgments}

The authors gratefully acknowledge the State Key Laboratory for Performance and Structure Safety of Petroleum Tubular Goods and Equipment Materials, CNPC Tubular Goods Research Institute, and China National Quality Supervision, Testing and Inspection Center of Oil Tubular Goods.

\section{References}

1. E.J. Omonbude, The transit oil and gas pipeline and the role of bargaining: a non-technical discussion, Energy Policy, 35, 6188 (2007).

2. L. Zhang, X. Li, C. Du, Effect of applied potentials on stress corrosion cracking of X70 pipeline steel in alkali solution, Materials \& Design, 30, 2259 (2009).

3. W.K. Kim, S.U. Koh, B.Y. Yang, Effect of environmental and metallurgical factors on hydrogen induced cracking of HSLA steels, Corrosion Science, 50, 3336 (2008).

4. J.L. Gonzalez, R. Ramirez, J.M. Hallen, Hydrogeninduced crack growth rate in steel plates exposed to sour environments, Corrosion, 53, 935 (1997).

5. B. Beidokhti, A.H. Koukabi, A. Dolati, Influences of titanium and manganese on high strength low alloy SAW weld metal properties, Materials Characterization, 60, 225 (2009).

6. S.S. Nayak, R.D.K. Misra, J. Hartmann, Microstructure and properties of low manganese and niobium containing HIC pipeline steel, Materials Science and Engineering: A, 494, 456 (2008).

7. G.T. Park, S.U. Koh, H.G. Jung, Effect of microstructure on the hydrogen trapping efficiency and hydrogen induced cracking of linepipe steel, Corrosion Science, 50, 1865 (2008).

8. X. Ren, W. Chu, J. Li, The effects of inclusions and second phase particles on hydrogen-induced blistering in iron, Materials Chemistry and Physics, 107, 231 (2008).

9. M. Elboujdaini, V.S. Sastri, J.R. Perumareddi, Studies on inhibition of hydrogen-induced cracking of linepipe steels, Corrosion, 62, 29 (2006).

10. V. Venegas, F. Caleyo, T. Baudin, Role of microtexture in the interaction and coalescence of hydrogen-induced cracks, Corrosion Science, 51, 1140 (2009).

11. C. Natividad, M. Salazar, A. Contreras, Sulfide stress cracking susceptibility of welded X-60 and X65 pipeline steels, Corrosion, 62, 375 (2006).

12. B. Beidokhti, A.H. Koukabi, A. Dolati, Effect of titanium addition on the microstructure and inclusion formation in submerged arc welded HSLA pipeline steel, Journal of Materials Processing Technology, 209, 4027 (2009).

13. B. Beidokhti, A. Dolati, A.H. Koukabi, Effects of alloying elements and microstructure on the susceptibility of the welded HSLA steel to hydrogen-induced cracking and sulfide stress cracking, Materials Science and Engineering: A, 507, 167 (2009).

14. M.A. Mansour, A.M. Alfantazi, M. El-boujdaini, Sulfide stress cracking resistance of API-X100 high strength low alloy steel, Materials \& Design, 30, 4088 (2009). 